\title{
Good quality epidemiological studies of oro-facial pain needed
}

\author{
What is the prevalence and associated risk factors of oro-facial pain (OFP)?
}

\begin{abstract}
Macfarlane TV, Glenny AM, Worthington HV. Systematic review of population-based epidemiological studies of oro-facial pain. J. Dent. 2001; 29(7):451-67
\end{abstract}

Data sources MEDLINE, Embase, Cinahl, Scientific Citation Index and Health CD databases. Reference lists of relevant and the journals Pain and "Community Dentistry and Oral Epidemiology" were handsearched.

Study selection Observational studies (cross-sectional surveys, cohorts case-control studies) and previous literature reviews of adult populations over 18 year of age were included. Oro-facial pain (OFP) was defined as pain located in the face, dental pain was excluded.

Data extraction and synthesis A standardised checklist was used to assess the methodological quality of each study by two reviewers before an attempt was made to summarise the results. The median quality score was $70 \%$ of the maximum attainable score. Due to methodological issues, it was not possible to pool the data on the prevalence of OFP.

Results 59 studies were evaluated. Age, gender and psychological factors were found to be associated with OFP, however there was not enough information on other factors such as local mechanical and comorbidities to draw any reliable conclusions. None of the factors fully fulfilled criteria for causality (Table 1).

Conclusions There is a need for good quality epidemiological studies of adequate sample size of OFP in the general population. To enable comprehensive examination of the aetiology of OFP, it is necessary to address a broad range of factors, eg demography and life-style, local mechanical factors, medical history and psychological factors. Data on

Table 1. Percentage of studies reporting factors which are possibly associated with OFP.

\begin{tabular}{lcc}
\hline & Cross-sectional & Cohort \\
\hline Age & 68 & 33 \\
Gender & 73 & 67 \\
Socio-economic characteristics & 24 & 50 \\
Education & 11 & 17 \\
Geographic area & 11 & -17 \\
Income/social class & 11 & - \\
Race/ethnicity & 5 & - \\
Marital status & 5 & 50 \\
Psychosocial factors & 11 & 17 \\
General state of health & 11 & 17 \\
Pain other than OFP & 16 & 50 \\
Headache & 24 & - \\
Denture status & 27 & 17 \\
Number of teeth & 19 & 33 \\
Parafunction & 14 & 17 \\
Joint clicking & 16 & 17 \\
Other & 16 & \\
\hline
\end{tabular}

Address for correspondence: TV Macfarlane, Turner Dental School, The University of Manchester, Higher Cambridge Street, Manchester M15 6FH, UK. E-mail: tatiana. macfarlane@man.ac.uk potential confounders and effect modifiers should also be collected and adjusted for in the statistical analysis.

\section{Commentary}

This systematic review had 4 main objectives; to describe and compare performance of several electronic data bases for identification of epidemiological studies on oro-facial pain (OFP); to identify and assess quality of population-based epidemiological studies of OFP; to determine the prevalence of OFP in different adult populations and to describe factors which are associated with OFP.

The review follows an excellent methodology for defining search strategy and initial inclusion criteria. The approach to determining quality is well defined with reported inter-examiner and intraexaminer levels of agreement. It is interesting to note that many of the check list items had low levels of agreement and less that half had good levels of agreement. Moderate agreement (kappa $=0.6$ ) between two observers for classification of papers into subgroups was reported. There was also a wide range in level of agreement between the two examiners for the component items of the quality checklists with more than half of the items having kappa values below 0.7 . This suggests that the validity of the quality assessment instrument is suspect, or calibration of the examiners was inadequate. Even the level of agreement between observers for classification of the studies by design was relatively low.

The first two objectives of this study were met. The study reported the sensitivity of electronic data bases in searching for epidemiological OFP studies. The quality of existing epidemiological and review articles were evaluated with an objective instrument.

However, the authors do not justify why they chose to include narrative reviews. Narrative reviews are subject to selection bias and therefore should not have been considered.

The final two stated objectives of this systematic review are not met. Unfortunately, although the authors completed a quality assessment on the selected articles, all articles were included in the results and ultimately the discussion. The authors should have planned a cut-off score below which the study would be rejected as being of inadequate quality. It is impossible to draw conclusions regarding prevalence of OFP in different populations and it is impossible to determine the factors associated with OFP from this data, when poor studies were not eliminated. The reader is left to attempt to judge the quality of the individual studies without adequate information on each specific study. Although according to the quality scores, some studies were of good quality, the good studies were not highlighted in this publication. Good evidence may exist for specific issues, but unfortunately the reader does not have the ability to interpret what specific evidence was properly collected and of good quality. There was a substantial time interval between completion of the search (1998) and acceptance for publication (2001) as a result this literature review is somewhat outdated. The abstract is misleading as the objective stated there was to "determine the prevalence and associated risk factors of orofacial pain", yet the bulk of the article addresses the overall quality of existing research. The result being that this study does not allow conclusions regarding the prevalence or risk factors and is only 
helpful in providing some general guidance for designing future epidemiological OFP research.

\section{Practice point}

- This study does not allow conclusions regarding the prevalence or risk factors.

\section{Paul W. Major}

Faculty of Medicine and Dentistry, University of Alberta, Edmonton, Canada

Evidence-Based Dentistry (2003) 4, 83-84.

doi:10.1038/sj.ebd.6400227

\begin{tabular}{l}
$\begin{array}{l}\text { Key to evidence graphic used in Evidence-based Dentistry with examples of study } \\
\text { designs for Therapy/prevention/aetiology and harm. Study designs for evidence levels } \\
\text { will be different for prognosis/diagnosis etc. (See Evidence-based Dentistry } \\
\text { 2003;4:p17-18) }\end{array}$ \\
\hline Evidence Graphic \\
\hline $3 \mathrm{~A}$
\end{tabular}

